\title{
Effects of self-monitoring of heart rate and additional sprint running on exercise intensity and technical performance during small-sided games in soccer
}

\author{
Paul Alan Swinton, Hamish Munro, Eimear Dolan, Katherine Burgess
}

\begin{abstract}
Objectives: The purpose of this study was to assess whether relatively simple interventions including self monitoring of heart rate and the use of targeted sprints for those with the lowest relative HR values $\left(\% \mathrm{HR}_{\max }\right)$ could be used to increase exercise intensity during small sided games (SSGs) in soccer. A secondary aim of the study was to assess the effect of these interventions on overall gameplay.

Design and Methods: Fourteen male semi-professional players performed SSGs (6 vs. 6) under four conditions including a control, a self-monitoring approach where players monitored their own HR via a wristwatch, and two sprint conditions where players with HR values below $90 \% \mathrm{HR}_{\max }$ performed sprints either during the game or during the recovery period between games. A linear mixed effects model was used to test for main effects whilst accounting for covariances between observations made on the same player.

Results: The results identified relatively small but significant differences in average $\% \mathrm{HR}_{\max }(\mathrm{p}<0.001)$ and $\mathrm{RPE}$ values $(\mathrm{p}<0.001)$ between the three modified conditions and the control. No significant differences were found between any of the modified conditions for measures of exercise intensity. On average, the modified conditions resulted in a $3.7 \%$ increase in $\% \mathrm{HR}_{\max }$ values and a $9.3 \%$ increase in RPE.

Conclusion: The results from this study demonstrate that exercise intensity of SSGs in soccer can be increased by relatively simple and practical manipulations, the most basic of which requires only the use of inexpensive HR monitors.

(Journal of Trainology 2016;5:53-60)
\end{abstract}

Key words: specific training $\mathbf{a}$ fitness $\mathbf{m}$ self regulation $\mathbf{a}$ football

\section{INTRODUCTION}

Small-sided games (SSGs) have become a popular training modality in soccer where condensed games are played with fewer players (i.e. 1 vs. 1 to 8 vs. 8) on reduced pitch areas. SSGs are frequently used as a means to concurrently develop technical ability, tactical ability and conditioning specific to the demands of the sport. ${ }^{1}$ Key elements in the study and implementation of SSGs include the monitoring and optimisation of exercise intensity. The most common measures used to quantify intensity during SSGs include heart rate (HR), blood lactate concentration, rating of perceived exertion (RPE) and GPS related information including total distance and distance covered in various speed zones. ${ }^{2}$ Whist some studies have employed multiple assessment types, ${ }^{3}$ monitoring HR remains the single most common measure used to assess intensity in both research and practice ${ }^{2}$. Analyses of the physiological demands of competitive soccer matches have revealed that average HR typically ranges between $80-90 \%$ of $\mathrm{HR}_{\text {max. }}{ }^{2}$ As a result, commonly the goal when performing SSGs is the attainment of exercise intensities of $90-95 \% \mathrm{HR}_{\max }$ in attempts to induce training adaptations that will transfer to competition. ${ }^{1}$ With junior soccer players, Impellizzeri et al. ${ }^{4}$ demonstrated that 12 weeks of training comprised of SSGs where players attained $90-95 \% \mathrm{HR}_{\max }$ for long periods resulted in improve- ments in $\dot{\mathrm{V}}_{2 \text { max }}$ and match performance indices (total distance covered and time spent during high-intensity running) similar to that obtained with standard aerobic interval training methods. Similar results demonstrating equivalent pre- to postimprovements in aerobic and intermittent tests after traditional exercise training or SSGs have been reported for youth soccer players and elite professionals provided exercise heart rate and total exercise time are comparable. ${ }^{5,6}$

The majority of research studies that have investigated SSGs in soccer have focused on modifications that can be made to increase exercise intensity. ${ }^{2}$ Common modifications include changes to pitch area, ${ }^{7}$ player number, ${ }^{8}$ removal of goalkeepers $^{9}$ and rule changes such as the number of ball touches permitted $^{10}$. Research has demonstrated that changes in pitch area and player number consistently influence exercise intensity, whereas most rule changes investigated have shown trivial or no effects. ${ }^{1,2}$ In general, the modifications described are expected to influence players relatively equally. However, one of the potential limitations with SSGs is that the fittest players with the highest $\dot{\mathrm{VO}}_{2 \max }$ may experience lower relative intensities and therefore reduced likelihood of training adaptations, ${ }^{2}$ As a result, Hoff et al. ${ }^{11}$ suggested that targeted modifications may be required to increase the exercise stimulus for those with the highest $\dot{\mathrm{V}} \mathrm{O}_{2 \max }$ and potentially reduce the exercise

Received August 2, 2016; accepted November 10, 2016

From the School of Health Sciences, Robert Gordon University, Aberdeen, UK (P.A.S., H.M., E.D., K.B.) and School of Physical Education and Sport, Universidade de Sao Paulo, Brazil (E.D.)

Communicated by Takashi Abe, PhD

Correspondence to Dr. Paul Alan Swinton, School of Health Sciences, Robert Gordon University, Aberdeen, UK. Email: p.swinton@rgu.ac.uk Journal of Trainology 2016;5:53-60 @ 2012 The Active Aging Research Center http://trainology.org/ 
stimulus for those with the lowest $\dot{\mathrm{V}} \mathrm{O}_{2 \max }$. Therefore, the purpose of this study was to investigate the effect of novel modifications to SSGs designed to create similar intensities across players with different fitness levels. Exercise intensity was monitored for each player using $\% \mathrm{HR}_{\max }$ values. Three modifications were investigated including a self monitoring approach and two forms of targeted additional sprint running where players with low $\% \mathrm{HR}_{\max }$ values performed sprints to increase exercise intensity. The three modifications investigated represented a progression of external involvement with respect to the performance support team, with self monitoring representing a player focused approach, whereas the additional sprint running approaches required involvement from the performance support team to manage real-time HR data and instruct players to perform sprints when necessary. It was hypothesised that each modification would increase exercise intensity as measured by average $\% \mathrm{HR}_{\max }$ compared with a control and that the sprint modifications that required greater external involvement would result in the largest increases in exercise intensity.

\section{METHODS \\ Experimental Approach to the Problem}

The study featured a cross sectional design with outcome measures used to assess intensity and technical actions across four different conditions applied to SSGs. The four conditions included: 1) a control condition (CON) featuring no modifications; 2) self monitoring (SM), where subjects were provided with a wrist watch displaying their HR and instructed to keep the value above $90 \%$ of their maximum; 3 ) after game shuttle (AGS), where players with an average $\mathrm{HR}$ below $90 \% \mathrm{HR}_{\max }$ performed a twenty yard shuttle comprising five yard separations during the intermission between games; and 4) in game sprint (IGS), where every 30 seconds the two players (one from each team) with the lowest $\% \mathrm{HR}_{\max }$ for the preceding interval performed a single return sprint to a marker located 10 yards behind their defending goal. Each condition was performed twice in a randomised order to increase the pool of data and to assess consistency. Data were collected over a 6-week period from August to September during the competition phase of the season.

\section{Subjects}

Fourteen male semi-professional soccer players (age $=25.9$ $\pm 2.7 \mathrm{yrs}$, mass $=78.6 \pm 4.7 \mathrm{~kg}$, height $=181.7 \pm 4.5 \mathrm{~cm}$, Yo-Yo $\mathrm{IR} 2$ distance $=830 \pm 32 \mathrm{~m}, \mathrm{HR}_{\max }=190.3 \pm 6.8 \mathrm{bpm}$ ) were recruited from a Scottish Junior North Super League team. The group consisted of five defenders ( 3 external and 2 central), five midfielders ( 2 external and 3 central) and four attackers. The players were selected by the head coach to create balance in ability and position between teams. Each player received an information sheet detailing the aims and objectives of the study as well as the potential risks involved. Prior to each testing session players completed a Physical Activity Readiness Questionnaire and provided written informed consent to be included. Approval for the study was obtained from the ethical review panel at the Robert Gordon University, Aberdeen, UK.

\section{Procedures}

Players began the study by performing the Yo-Yo-IR2 test in order to obtain a measurement of each individual's maximum HR. ${ }^{12}$ The uppermost HR value recorded throughout the test was selected as the subjects $\mathrm{HR}_{\max } \cdot{ }^{5}$ Eight separate testing sessions (two for each of the four conditions) each comprising a series of SSGs were then performed with approximately three to five days recovery allocated between sessions. Testing commenced at the same time of day in order to limit the effects of circadian variations on HR data. Testing was carried out at the beginning of the Subjects' soccer training sessions to avoid the effects of fatigue from prior exertions. Testing sessions were conducted indoor on an artificial $4^{\text {th }}$ generation surface. Each testing session commenced with the same standardised warmup, followed by 3 x 6-minute SSGs with a 2-minute intermission between games. Game formats of 6 vs. 6 (including goal keepers) played on a $40 \times 30$ yard pitch were based on those implemented by Little and Williams ${ }^{13}$ which demonstrated high reliability in outcome measures. The sequence in which conditions were allocated across the eight testing sessions was randomised to eliminate order effects.

\section{Control Condition (CON)}

No modifications out with standard soccer rules were placed on the games (i.e. players could take any number of touches and score from anywhere) with standard rules also applied to throw-ins and corner kicks. The offside rule was not enforced as is customary during SSGs. Players were instructed to maintain as high an intensity as possible. The modified conditions assessed in other testing sessions followed the same game rules as the control but comprised additional instructions or requirements according to the specific condition.

\section{Self Monitoring Condition (SM)}

Players wore a polar wristwatch FT1 (Polar Electro Oy, Kempele, Finland) that displayed their own HR via transmission from an individually worn chest strap. Each player was informed of the HR value that equalled $90 \%$ of their maximum and were instructed to attempt to maintain their HR above this threshold. Players were instructed to monitor their wristwatch display during natural breaks in the game (i.e. throw-ins, corner kicks, goals and slow passages of play).

\section{After Game Shuttle Condition (AGS)}

The average HR for each player was calculated immediately after each game. Players with average HR values below $90 \%$ of their maximum were then instructed to perform a 20 yard shuttle with 5 yard separations (i.e. 5 yards sprint and return to the start, 10 yards sprint and return to the start, 15 yards sprint and return to the start line and 20 yards sprint returning to the start, for a total of 100 yards). Shuttles were performed during the 2 minute intermission between games 1 and 2 or immediately after the final game.

\section{In Game Sprint Condition (IGS)}

The IGS condition used live-streaming analysis to determine which two players (one from each team) had the lowest aver- 
age $\% \mathrm{HR}_{\max }$ during $30 \mathrm{~s}$ intervals. The two players with the lowest $\% \mathrm{HR}_{\max }$ values were instructed to perform a return sprint around a marker set 10 yards behind their defending goal and then return to play. No sprints were allocated to a team if HR values for all players were above the threshold for the interval, creating potential situations where no players or only one player had to perform the action. Sprints were conducted every $30 \mathrm{~s}$, excluding the first $30 \mathrm{~s}$ of each game to enable players to raise their HR.

\section{Outcome measures}

HR was measured on out-field players only using a shortrange telemetry Polar Team ${ }^{2}$ Pro System (Polar Electro OY, Kempele, Finland) with data collected at a frequency of $1 \mathrm{~Hz}$. Following each testing session, HR data was transformed into a percentage of each subjects $\mathrm{HR}_{\max }$ and analysis conducted using average values obtained across the games only (i.e. HR data collected during the intermission was not included in the final analysis). Further analysis of the HR data was based on the percentage of time spent in five HR zones, including $50-59 \% \mathrm{HR}_{\max }=$ zone $1,60-69 \%=$ zone $2,70-79 \% \mathrm{HR}_{\max }=$ zone $3,80-89 \% \mathrm{HR}_{\max }=$ zone 4 and $\geq 90 \% \mathrm{HR}_{\max }=$ zone 5 . Subjective assessment of exercise intensity was collected using the 10-point RPE scale proposed by Foster et al. ${ }^{14}$ RPE values were obtained immediately after each game and prior to sprints if they were to be performed. All subjects were familiarised with the scale prior to commencement of the study.

Assessment of technical aspects of each game were obtained by notational analysis of video footage. Three fixed digital video cameras (Panasonic, SDR-S15, Osaka, Japan) were included with one video camera positioned perpendicular to the long axis of the pitch along the halfway line and the remaining two video cameras positioned along the long axis behind each goal. All cameras were positioned at a distance of $20 \mathrm{~m}$ from the pitch edge and at an elevation of $5 \mathrm{~m}$ to provide adequate coverage. A notational analysis system was used to obtain thirteen discrete gameplay actions (defined in table 1). The selected actions provided information regarding the quality of the match in addition to the type of tactics employed. ${ }^{5}$

\section{Statistical Analysis}

Data are presented as mean \pm SDs. Percentage of $H R_{\max }$ and RPE data were collected for the three games played under each condition on two separate occasions. Data collected within each outcome measure were combined and the effects of condition, game number and testing occasion (1 or 2) were assessed by fitting linear models with random effects to account for the covariance between observations made on the same player. Main effects and interaction effects were assessed using generalized likelihood ratio tests and the appropriate standard chi-squared asymptotic reference distribution. The time spent in each HR zone was averaged over the three games played within a testing session and therefore the main effects of condition and testing occasion only were assessed for this outcome measure. Statistical analyses were conducted using the lme4 package ${ }^{15}$ in the statistical environment R (R Core Team). $\mathrm{P}$ values for fixed parameter estimates were calculated using t-tests and Satterthwaite's approximation for degrees of freedom with the lmerTest library in R. ${ }^{16}$ Local effect sizes were obtained for $\% \mathrm{HR}_{\max }$ and $\mathrm{RPE}$ values by calculating pro-

Table 1 Summary of technical variables recorded

\begin{tabular}{|c|c|}
\hline Technical Action & Definition \\
\hline Total Passes & $\begin{array}{l}\text { Number of times per game that a player sends the ball to a teammate (e.g. using the foot, thigh or chest; } \\
\text { using various techniques such as ground, lofted, chip, flick or volley; over short or long distances). }\end{array}$ \\
\hline$\%$ Successful Passes & The $\%$ of passes that reached a player of the same team. \\
\hline$\%$ Forward passes & The $\%$ of passes that were played towards the opposition goal \\
\hline$\%$ Forward pass success & The $\%$ of passes that reached a player of the same team that was played towards the opposition goal. \\
\hline Ball possessions & The number of times ball possession is turned over between the two teams. \\
\hline Passes Per Possession & The number of passes completed per possession. \\
\hline Dribbles & Player in possession, with ball at feet, runs with ball, beats or attempts to beat an opponent. \\
\hline Tackles & An action intending to dispossess an opponent who is in possession of the ball. \\
\hline Shots & Player in possession meaningfully sends the ball towards the goal in an attempt to score. \\
\hline$\%$ Shots on Target & The $\%$ of shots that are within the frame of the goalposts. \\
\hline Goals & The number of times the ball crosses the goal line, between the goal posts without a prior infringement. \\
\hline Lost Balls & The number of times the ball exits the pitch perimeter. \\
\hline Interceptions & The number of times a player contacts or stops the ball from reaching its intended target. \\
\hline
\end{tabular}


portional reduction in variance using the level-1 residual variance estimated in the base model and with predictor added. ${ }^{17}$

\section{RESULTS}

No significant effects of testing occasion were found for any of the models fitted ( $p=0.244$ to 0.9243 ), thereby indicating that outcome measures remained consistent across the repeated sessions. Percentage $\mathrm{HR}_{\max }$ data are illustrated in figure 1. Significant main effects were obtained for condition $\left(\chi_{3}^{2}=\right.$ $40.37, \mathrm{p}<0.001)$ and game number $\left(\chi_{2}^{2}=23.75, \mathrm{p}<0.001\right)$ In addition, a greater effect size was obtained for condition in comparison to game number (proportional reduction in variance: $13.5 \%$ and $7.6 \%$, respectively). Analysis of fixed effects revealed that modified conditions produced significantly greater $\% \mathrm{HR}_{\max }$ values than those observed during the control $\left(\mathrm{t}_{220.94}=4.65, \mathrm{p}<0.001\right.$ to $\left.\mathrm{t}_{222.49}=5.989, \mathrm{p}<0.001\right)$. In contrast, no significant pairwise differences were observed between any of the modified conditions $\left(\mathrm{t}_{221.41}=1.437, \mathrm{p}=\right.$ 0.152 to $\left.t_{221.22}=0.485, p=0.628\right)$. In particular, the modified conditions increased $\% \mathrm{HR}_{\max }$ on average by approximately $4 \%$. A significant interaction effect between game number and condition $\left(\chi_{4}^{2}=13.24, \mathrm{p}=0.039\right)$ was also obtained with a consistent rise in $\% \mathrm{HR}_{\max }$ values observed across games for the control condition only (Figure 1).

RPE data followed a similar pattern to that observed for $\% \mathrm{HR}_{\max }$ values (Figure 2 ) with significant main effects found for condition $\left(\chi_{3}^{2}=25.74, \mathrm{p}<0.001\right)$ and game number $\left(\chi_{2}^{2}=\right.$ $45.959, \mathrm{p}<0.001)$ However, for RPE values a larger effect size was obtained for game number in comparison to condition (proportional reduction in variance: $16.1 \%$ and $7.1 \%$, respectively). The fixed effects parameters demonstrated that each of the modified conditions produced significantly greater RPE values than those observed during the control $\left(\mathrm{t}_{225.83}=3.195\right.$, $\mathrm{p}=0.0016$ to $\left.\mathrm{t}_{224.26}=4.459, \mathrm{p}<0.001\right)$, with no significant pairwise differences found between modified conditions $\left(\mathrm{t}_{224.48}=1.249, \mathrm{p}=0.213\right.$ to $\left.\mathrm{t}_{222.77}=0.063, \mathrm{p}=0.950\right)$. In particular, the modified conditions increased RPE on average by approximately 8 to $10 \%$. A significant interaction effect between game number and condition was also obtained $\left(\chi_{4}^{2}=18.677, \mathrm{p}=0.047\right)$. The descriptive data shows that RPE values increased across the three games for all conditions, however, the rate of increase appeared greater during the control.

The percentage of time spent in each of the five HR zones was averaged over the three games played during each testing session and is presented in figure 3. A significant main effect of condition was obtained for time spent in the three lowest HR zones $\left(\chi_{3}^{2}=9.243, \mathrm{p}<0.001\right.$ to $\left.\chi_{3}^{2}=25.652, \mathrm{p}=0.026\right)$. The model fixed effects revealed that the percentage time spent in HR zones 1 to 3 was significantly less for each of the three modified conditions compared with the control $\left(\mathrm{t}_{63.61}=\right.$ $-4.925, \mathrm{p}<0.001$ to $\left.\mathrm{t}_{63.22}=-2.058, \mathrm{p}=0.044\right)$. Conversely, no significant main effect of condition was obtained for the percentage time spent in the two highest HR zones $\left(\chi_{3}^{2}=3.220\right.$ to $6.05, \mathrm{p}=0.359$ to $\mathrm{p}=0.108$ ).

Analysis of technical variables revealed consistent values for all variables across conditions except the percentage of forward passes, where a significant main effect of condition was obtained $\left(\chi_{3}^{2}=24.2, \mathrm{p}<0.001\right.$, Table 2$)$. The results demonstrated that the proportion of forward passes was significantly greater during the IGS condition compared to all others $\left(\mathrm{t}_{63.21}=5.905, \mathrm{p}<0.001\right.$ to $\left.\mathrm{t} 63.24=4.958, \mathrm{p}<0.001\right)$.

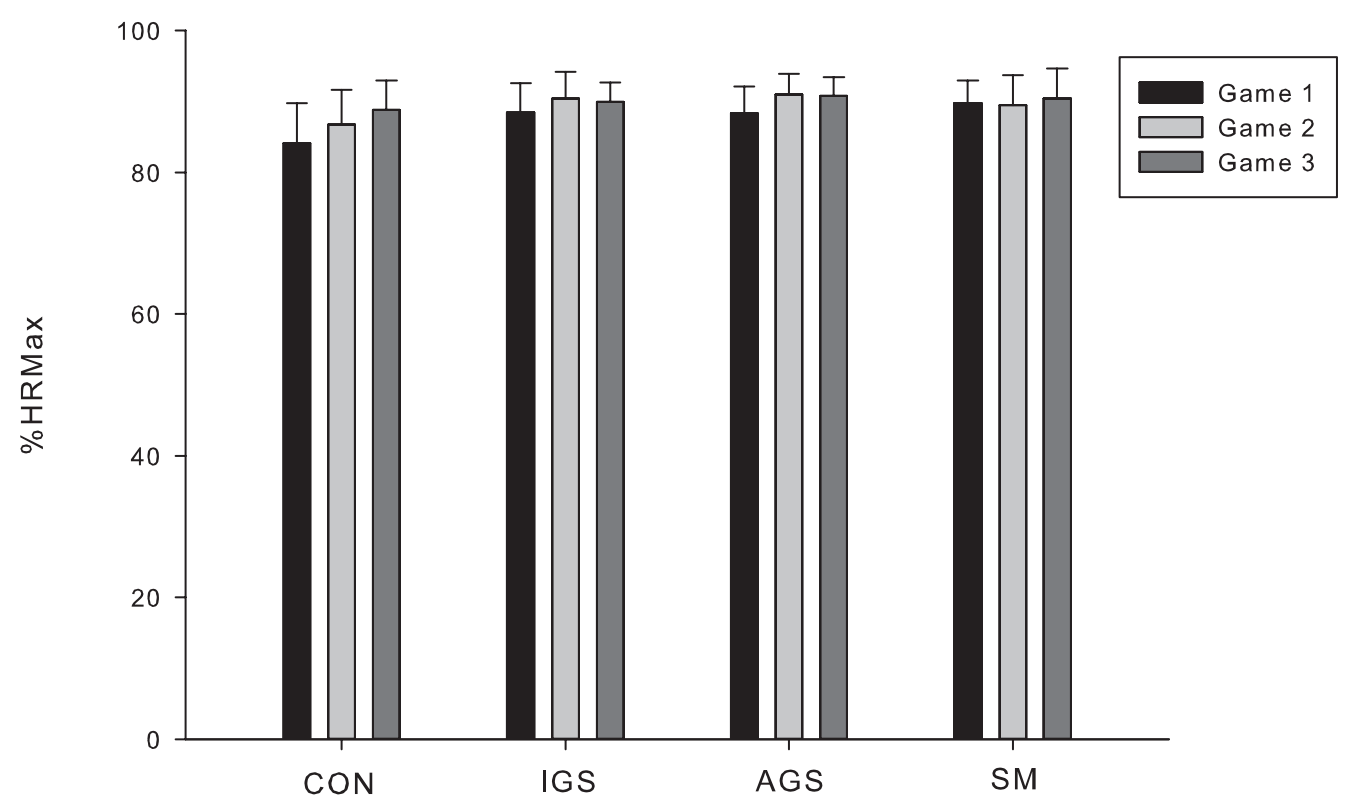

Figure 1 Mean \pm SDs for percentage of maximum heart rate $\left(\% H R_{\max }\right)$ across games and conditions

AGS = after game shuttle, $C O N=$ control, IGS = In game sprint and SM = self monitoring. Significant main effects of condition $(p<0.001)$ and game number were obtained $(p<0.001)$, as well as a significant interaction effect between condition and game number $(p=0.039)$. 
Table 2 Mean \pm SDs for technical variables averaged across the three games for each condition

\begin{tabular}{lcccc}
\hline & CON & AGS & IGS & SM \\
\hline Total Passes & $99.0 \pm 9.6$ & $100.0 \pm 12.0$ & $101.0 \pm 17.1$ & $105.8 \pm 10.9$ \\
\%Successful Passes & $86.1 \pm 5.0$ & $85.5 \pm 4.4$ & $85.7 \pm 2.6$ & $88.8 \pm 5.4$ \\
\%Forward passes & $57.0 \pm 4.2$ & $58.9 \pm 4.8$ & $69.8 \pm 4.8^{*}$ & $62.2 \pm 3.5$ \\
\%Forward pass success & $87.0 \pm 2.4$ & $83.9 \pm 5.4$ & $86.4 \pm 3.7$ & $85.2 \pm 5.6$ \\
Ball possessions & $27.2 \pm 3.4$ & $26.5 \pm 3.3$ & $29.2 \pm 3.2$ & $26.0 \pm 3.1$ \\
Passes Per Possession & $3.9 \pm 0.7$ & $3.8 \pm 0.8$ & $3.6 \pm 0.6$ & $4.2 \pm 0.8$ \\
Dribbles & $23.5 \pm 3.9$ & $26.0 \pm 5.6$ & $26.0 \pm 3.2$ & $25.7 \pm 3.3$ \\
Tackles & $6.3 \pm 2.5$ & $4.7 \pm 1.0$ & $4.5 \pm 2.1$ & $3.7 \pm 1.8$ \\
Shots & $11.2 \pm 2.3$ & $10.5 \pm 3.2$ & $13.5 \pm 3.3$ & $12.2 \pm 3.9$ \\
\% Shots on Target & $58.3 \pm 14.4$ & $62.4 \pm 13.8$ & $63.7 \pm 19.8$ & $63.4 \pm 23.0$ \\
Goals & $3.7 \pm 1.9$ & $3.3 \pm 1.0$ & $3.8 \pm 1.8$ & $3.3 \pm 1.4$ \\
Lost Balls & $10.5 \pm 2.2$ & $9.8 \pm 1.7$ & $11.5 \pm 2.7$ & $10.2 \pm 2.1$ \\
Interceptions & $11.5 \pm 2.7$ & $14.7 \pm 2.3$ & $14.2 \pm 4.2$ & $14.3 \pm 4.4$ \\
\hline
\end{tabular}

AGS $=$ After-game shuttle, $\mathrm{CON}=$ Control, IGS $=$ In-game sprint and $\mathrm{SM}=$ Self-monitoring. $*$ Significantly different from all other conditions $(\mathrm{p}<0.001)$

\section{DISCUSSION}

The primary aim of the study was to test the hypothesis that relatively simple and practical modifications including self monitoring of heart rate and inclusion of targeted additional sprints could be used to increase exercise intensity of SSGs in soccer. The results obtained confirm this hypothesis and demonstrate that the modifications investigated produce relatively small but significant increases in HR values. Additional support for this hypothesis was obtained from RPE data demonstrating that player's perceptions of the exercise intensity also increased when modifications were included. In contrast, the hypothesis that the sprint modifications that required greater external involvement from performance support staff would result in the largest increases in exercise intensity was not supported, with no significant differences found between the modified conditions for any of the outcome variables measured. A secondary aim of the present study was to monitor technical actions associated with each of the conditions to assess whether the modifications altered overall gameplay. Importantly, successful modifications to SSGs should have the potential to increase exercise intensity whilst not being counterproductive with respect to technical gameplay. The results demonstrated that only one of the technical actions monitored was affected by the modifications investigated. Specifically, the IGS condition resulted in a significantly greater percentage of forward passes compared to all other conditions, with a $12.8 \%$ increase observed relative to the control. During the IGS condition there were frequent periods of reduced player numbers due to players performing sprints and at times this may have occurred only for one team resulting in 5 vs. 4 situations. Teams are frequently trained to counter attack and exploit numerical advantages explaining the increased percentage of forward passes obtained with this modification. ${ }^{10}$

When HR values were averaged across the three games per- formed in a testing session the group mean $\% \mathrm{HR}_{\max }$ for the control condition was $86.6 \%$. This value is consistent with previous research that has reported average $\% \mathrm{HR}_{\max }$ values ranging from 82.8 to $87.5 \%$ for 6 vs. 6 games with similar pitch dimensions. ${ }^{8,13,18}$ The AGS, IGS and SM modifications compared to the control resulted in a 3.8, 3.5, and 3.7\% increase in $\% \mathrm{HR}_{\max }$, respectively. The $\% \mathrm{HR}_{\max }$ values were relatively consistent across players and after controlling for the correlated values with a mixed effects model the relatively small differences were shown to be statistically significant providing strong evidence that the increases were not due to random variation. Previous studies investigating modifications in terms of player number, pitch size and rule changes have reported similar changes in $\% \mathrm{HR}_{\max }$ values to those reported here. Manolopoulos et al. ${ }^{19}$ with elite Greek professional soccer players reported increases of $2.8,3.8$ and $5.4 \%$ in $\% \mathrm{HR}_{\max }$ when moving from 7 vs. 7 to 6 vs. 6,5 vs. 5 and 4 vs. 4 games, respectively. Similarly, whilst investigating eight different combinations of SSGs with professional English soccer players including manipulations to player number ( 2 vs. 2 to 8 vs. $8)$, pitch dimension $(30 \times 20$ to $70 \times 45 \mathrm{yds})$ and game structure $(4 \times 2 \mathrm{~min}$ to $3 \times 10 \mathrm{~min})$, Little and Williams ${ }^{13}$ reported that mean $\% \mathrm{HR}_{\max }$ values were consistent and ranged from $87-91 \%$. Therefore, although the percentage differences observed in the present study appear relatively small, they are in line with other manipulations that have been considered to increase exercise intensity during SSGs. Similarly, calculation of the effect size for condition revealed a relatively small effect with the addition of condition resulting in a $13.5 \%$ reduction in the within-subject random error compared to the intercept only model. However, a larger effect was obtained for condition compared with game number $(7.6 \%$ reduction in the within-subject random error), indicating that the relatively small effect obtained with the modification may be of practical 


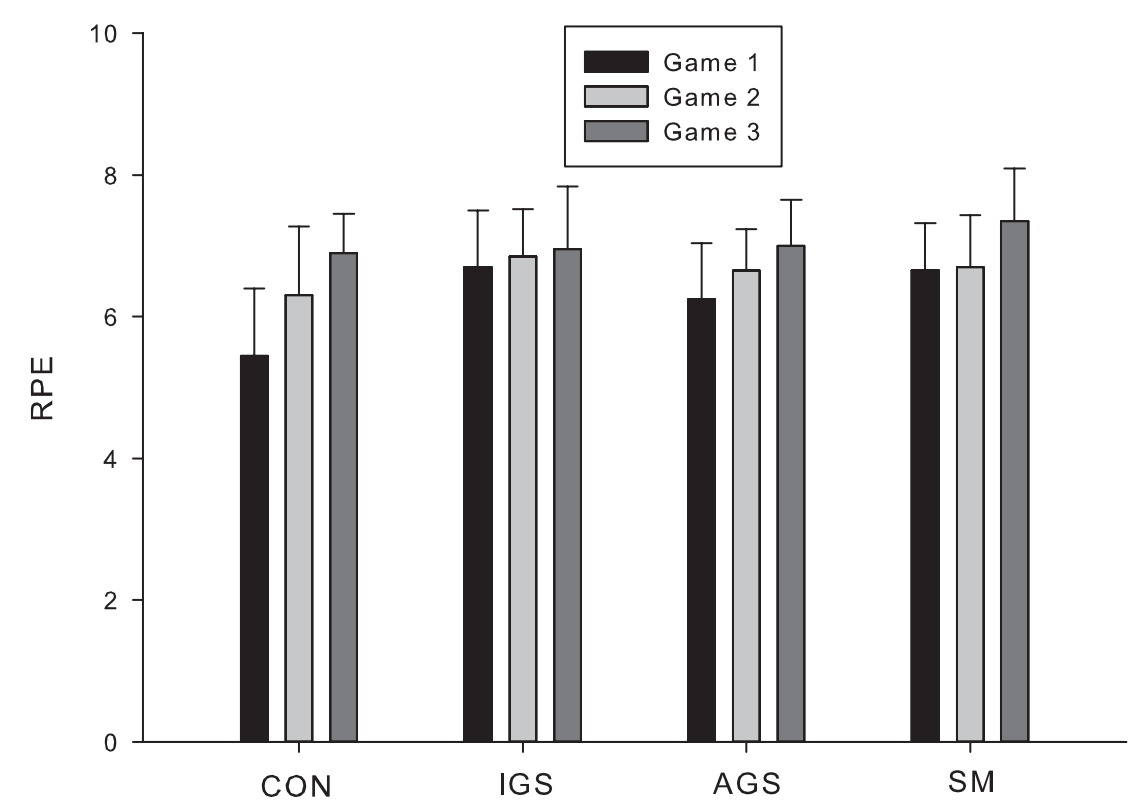

Figure 2 Mean \pm SDs for rating of perceived exertion (RPE) across games and conditions.

AGS = after game shuttle, CON = control, IGS = In game sprint, and SM = self monitoring. Significant main effects of condition $(p<0.001)$ and game number were obtained $(p<0.001)$, as well as a significant interaction effect between condition and game number $(p=0.047)$.

significance. Importantly, relatively small percentage increases in exercise intensity may accumulate to augment adaptations due to the frequent use of SSGs as a training modality in soccer. $^{11}$

Results from RPE data collected in the present study followed a similar profile to that obtained for $\% \mathrm{HR}_{\max }$. The overall mean RPE score for the present study was $6.8 \pm 0.9$ (range $4-9$ ) indicating that the player's perception of the difficulty of the sessions was typically between hard and very hard. ${ }^{14}$ The lowest mean RPE values were recorded during the control $(6.2 \pm 0.7)$ and were approximately $8-10 \%$ higher during the modified conditions (Figure 2). Previous research has established that RPE can be implemented with SSGs to accurately reflect exercise intensity ${ }^{20}$ Due to variations in RPE scales used in the literature and the many different SSG formats featured it is difficult to compare results across studies. Those that have applied the Borg CR-10 RPE scale have reported mean values to range between $7.0-7.7 .5,20$ The higher mean RPE values reported in previous studies may be related to differences in game format. Coutts et al. ${ }^{20}$ and Dellal et al. ${ }^{5}$ employed smaller player games including 3 vs. 3 formats. Previous research has established that SSGs featuring smaller numbers of players result in a greater number of anaerobic actions being performed, ${ }^{2}$ which may lead players to perceive a more intense training stimulus. Interestingly, the highest RPE values obtained in the present study occurred during the SM condition. During the final game played under each condition the mean RPE was less than or equal to 7 for all other conditions, whilst the mean RPE during SM was equal to 7.4. It is possible that these high values were partly due to the biofeedback provided by the wrist watches and the players being objectively aware that their HR was close to maximum.
Researchers and practitioners frequently analyse time spent in various HR zones in attempts to quantify the training stimulus. In particular, researchers have focused on the $90-100 \%$ HR zone due to many regarding this intensity as optimal to create physiological adaptations relevant to performance in soccer. ${ }^{11,13}$ In the present study the percentage time spent in five different HR zones was analysed. The results showed that under the modified conditions the percentage time accounted for by the three lowest $\mathrm{HR}$ zones $\left(50-79 \% \mathrm{HR}_{\max }\right.$, Figure 3) was significantly lower compared with the control. Surprisingly, no significant main effect of condition was obtained for percentage time spent in the two highest HR zones $\left(80-100 \% \mathrm{HR}_{\max }\right)$. Although mean percentage times were higher for each of the three modified conditions (Figure 3 ), the variation observed between players was greater than the variation observed for other outcome measures and it is likely the study was underpowered for these measurements and therefore unable to identify the true underlying effect. Similar large standard deviations were reported by Owen et al. ${ }^{21}$ when investigating time spent in HR zones suggesting that this outcome measure can encompass greater between subject variation compared with $\% \mathrm{HR}_{\max }$ and $\mathrm{RPE}$. Variation in player response may be due to multiple factors including positional differences and player behaviour with regards to off the ball movements. Variability is influenced by selection of the endpoints for each zone and it is likely that a reduced number of wider HR zones would reduce variability.

In addition to main effects obtained for condition, models fitted for $\% \mathrm{HR}_{\max }$ and $\mathrm{RPE}$ identified a significant main effect of game number and a significant interaction effect between condition and game number. In particular, the fixed effect parameters representing each of the games showed that 


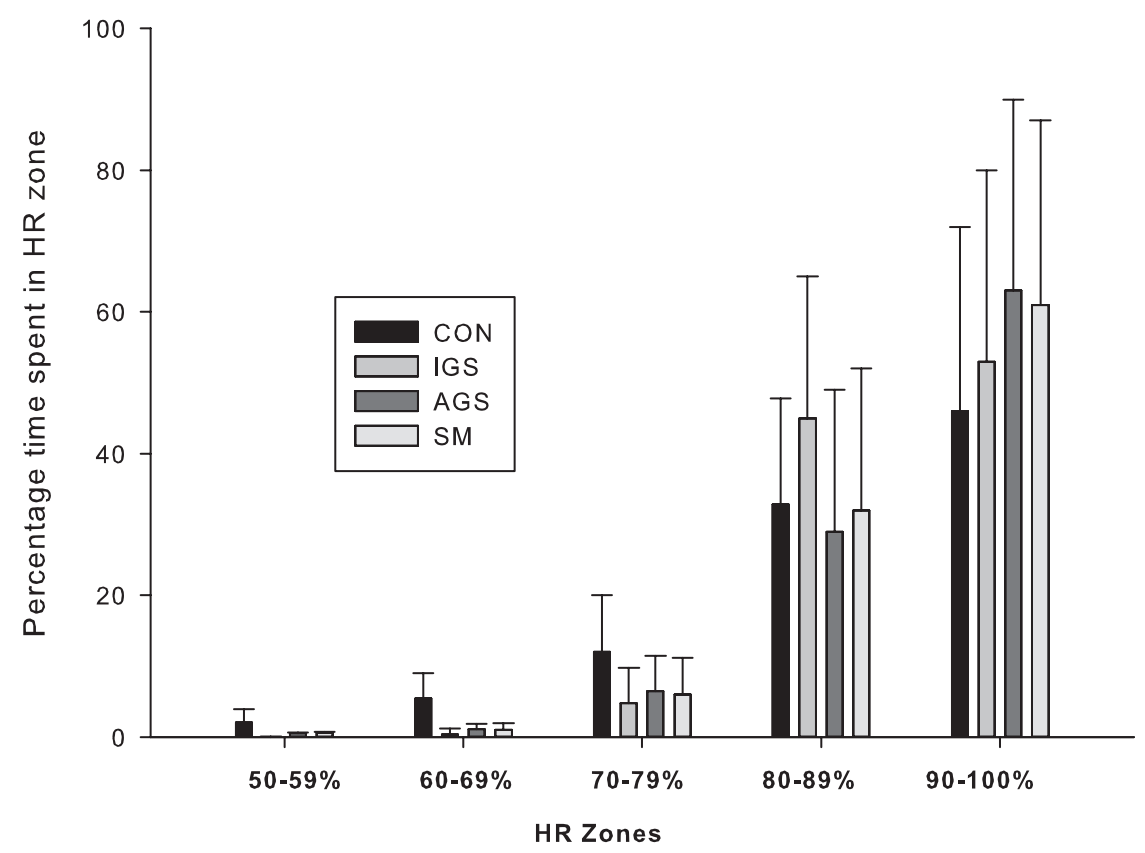

Figure 3 Mean \pm SDs for percentage of time spent in five different HR zones across conditions.

AGS = after game shuttle, CON = control, IGS = In game sprint, and SM = self monitoring. Significant main effects of condition were obtained for HR Zones 50-59\% ( $<<0.001), 60-69 \%(p<0.001)$ and $70-79 \%(p=0.026)$.

$\% \mathrm{HR}_{\max }$ increased significantly between game 1 and game 2 $(\mathrm{p}<0.001)$ and then plateaued. In contrast, significant increases were obtained between all games $(p<0.001)$ for RPE values. Increases in $\% \mathrm{HR}_{\max }$ between games may be explained by the intermission providing insufficient time for HR to fully recover to resting values and therefore proceeding games begin at higher values. In addition, accumulation of fatigue and metabolic by-products from prior games has been proposed to increase $\% \mathrm{HR}_{\max }$ values and perceived intensity of the exercise stimulus. ${ }^{4}$ These HR related effects may be less pronounced as values progress towards their maximum, hence explaining the plateau recorded between games 2 and 3 .

The significant interaction effect noted between condition and game number can be explained by the different pattern of increase in $\% \mathrm{HR}_{\max }$ and $\mathrm{RPE}$ values across games between the control and modified conditions. During the control the increase in $\% \mathrm{HR}_{\max }$ and $\mathrm{RPE}$ between games was relatively large and consistent. However, the same relatively large and consistent increases were not seen for all of the modified conditions. The disparity in response across games is likely due in part to the finding that the largest differences between the control and modified conditions occurred during game 1. That is, the largest effect of the modified conditions appears to be increasing the immediate exercise intensity associated with SSGs. This was to be expected during the IGS condition as those with lower HR values were provided with additional high intensity work that would be expected to instantaneously increase HR in a somewhat-linear manner. ${ }^{22}$ However, similar results from the SM and AGS conditions suggest that feedback related to target intensities and anticipatory behaviours, respectively, can be used to increase the immediate exercise intensity in SSG. A number of previous studies have demon- strated that biofeedback of HR values can be used by individuals to achieve and maintain target exercise intensities. ${ }^{23,24}$ However, previous studies have been conducted in more closed environments using cycle ergometers ${ }^{24}$ and treadmills ${ }^{23}$ where intensity is easier to control. The results from the present study demonstrate that athletes can use biofeedback to better maintain target intensities in more dynamic sport-specific environments. Finally, the observation that the AGS condition resulted in significantly greater $\% \mathrm{HR}_{\max }$ values compared to control in game 1 (prior to any additional sprints being conducted), suggests the presence of an anticipatory behaviour or potentially response to a motivator. External regulation through punishments can be viewed as a common but controversial coaching practice that may attenuate players intrinsic motivation ${ }^{25}$ and has been shown to reduce participation levels in longitudinal studies ${ }^{26}$. However, in the context of the present study where subjects were high level enthusiastic athletes it is unclear how performance of sprints related to missing personal targets was perceived. As a result, further research to explore the motivational and anticipatory behaviours associated with additional targeted exercise in similar athletes is required.

\section{Practical Applications}

In conclusion, this study demonstrates that the exercise intensity of SSGs in soccer can be increased by relatively simple and practical manipulations that can be implemented with resources common to amateur and professional sport teams. Importantly, the study also demonstrates that the manipulations do not negatively alter the technical aspects of the games. Therefore, it is recommended that soccer teams currently employing SSGs as part of their conditioning regime incorporate the modifications reviewed in this study. In particular, it is 
recommended that coaches always provide players with wristwatches to monitor their own HR with respect to target intensities that may be set on an individual basis. Additionally, it is recommended that coaches employ targeted additional sprints as part of a periodized program. Factors such as the threshold to induce sprints and distance covered may be modified by coaches in attempts to further manipulate exercise intensity. Further research incorporating positional tracking such as GPS may elucidate how the modifications increased exercise intensity, particularly in the early stages of games. In addition, further research is required to better understand anticipatory behaviours and motivational aspects of assigning additional high intensity work to athletes based on missing exercise targets.

\section{REFERENCES}

1. Halouani J, Chtourou H, Gabbett T, et al. Small-sided games in team sports training: A brief review. J Strength Cond Res 2014; 28: 3594-3618.

2. Hill-Haas SV, Dawson B, Impellizzeri FM et al. Physiology of small-sided games training in football: A systematic review. Sports Med 2011; 41: 199220.

3. Hill-Haas S, Coutts A, Rowsell G et al. Variability of acute physiological responses and performance profiles of youth soccer players in small-sided games. J Sci Med Sport 2008; 11: 487-490.

4. Impellizzeri FM, Marcora SM, Castagna $\mathrm{C}$ et al. Physiological and performance effects of generic versus specific aerobic training in soccer players. Int J Sports Med 2006; 27: 483-492.

5. Dellal A, Varliette C, Owen A et al. Small sided games versus interval training in amateur soccer players: Effects on the aerobic capacity and ability to perform intermittent exercises with changes of direction. $J$ Strength Cond Res 2012; 26: 2712-2720.

6. Reilly T, White C. Small-sided games as an alternative to interval-training for soccer players [abstract]. J Sports Sci, 2004; 22: 559

7. Casamichana D, Castellano J. Time-motion, heart rate, perceptual and motor behaviour demands in small-sides soccer games: Effects of pitch size. J Sports Sci 2010; 28: 1615-1623.

8. Katis A, Kellis E. Effects of small-sided games on physical conditioning and performance in young soccer players. J Sports Sci 2009; 8: 374-380.

9. Mallo J, Navarro E. Physical load imposed on soccer players during smallsided training games. J Sports Med Phys Fitness 2008; 48: 166-171.

10. Dellal A, Lago-Penas C, Wong del P et al. Effect of the number of ball contacts within bouts of 4 vs. 4 small-sided soccer games. Int J Sports
Physiol Perform 2011; 6: 322-333.

11. Hoff J, Wisloff U, Engen LC et al. Soccer specific aerobic endurance training. Br J Sports Med 2002; 36: 218-221.

12. Krustrup P, Mohr M, Amstrup T et al. The yo-yo intermittent recovery test: Physiological response, reliability, and validity. Med Sci Sports Exerc 2003; 35: 697-705.

13. Little T, Williams A. Suitability of soccer training drills for endurance training. J Strength Cond Res 2006; 20: 316-319.

14. Foster C, Florhaug JA, Franklin J et al. A new approach to monitoring exercise training. J Strength Cond Res 2001; 15: 109-115.

15. Bates DM, Machler M, Bolker et al. (2014). Fitting linear mixed-effects models using lme4. Journal of Statistical Software, http://arxiv.org/abs/ 1406.5823 .

16. Kuznetsova A, Brockhoff PB, Christensen RH. Package "ImerTest":tests for random and fixed effects for linear mixed effect models (lmer objects of lme4 package) R Package version 2.0-3, 2013.

17. Peugh P. A practical guide to multilevel modeling. J School Psychol 2010; 48: 85-112.

18. Rampinini E, Impellizzeri FM, Castagna C et al. Factors influencing physiological responses to small-sided soccer games. J Sports Sci 2007; 25(6), 659-666

19. Manolopoulos E, Kalapotharakos VI, Ziogas G et al. Heart rate responses during small-sided soccer games. J Sports Med Doping Stud 2012; 2: 36-39.

20. Coutts AJ, Rampinini E, Marcora SM et al. Heart rate and blood lactate correlates of perceived exertion during small-sided soccer games. $J$ Science Med Sport 2009; 12: 79-84.

21. Owen AL, Wong DP, McKenna M et al. Heart rate responses and technical comparison between small- vs. large-sided games in elite professional soccer. J Strength Cond Res 2011; 25: 2104-2110.

22. Drust B, Reilly T, Cable NT. Physiological responses to laboratory-based soccer-specific intermittent and continuous exercise. J Sports Sci 2000; 18: 885-892.

23. Moleiro MA, Cid FV. (2001). Effects of biofeedback training on voluntary heart rate control during dynamic exercise. Appl Psychophysiol Biofeedback 2001; 26: 279-292.

24. Shaykevich A, Grove RJ, Jackson B et al. Auditory feedback improves heart rate moderation during moderate-intensity exercise. Med Sci Sports Exerc 2015; 47: 1046-1051.

25. Weiss MR, Amorose AJ. Motivational Orientations and Sport Behavior. In Advances in Sport Psychology. (edited by T.S. Horn), 3rd edn. pp.115-155. Champaign, IL: Human Kinetics, 2008.

26. Smith DJ. A framework for understanding the training process leading to elite performance. Sports Med 2003; 33: 1103-1126. 